MATEMATIKA, 2017, Volume 33, Number 2, 241-246

(C) Penerbit UTM Press. All rights reserved

\title{
Fuzzy L-closed sets
}

\author{
Talal Al-Hawary \\ Yarmouk University \\ Department of Mathematics, Irbid-Jordan \\ e-mail: talalhawary@yahoo.com
}

\begin{abstract}
Our goal in this paper is to introduce the relatively new notions of fuzzy L-closed and fuzzy L-generalized closed sets. Several properties and connections to other well-known weak and strong fuzzy closed sets are discussed. Fuzzy L-generalized continuous and fuzzy L-generalized irresolute functions and their basic properties and relations to other fuzzy continuities are explored.
\end{abstract}

Keywords Fuzzy L-open set; fuzzy L-closed set; fuzzy L-generalized closed set; fuzzy L-generalized continuous function.

AMS mathematics subject classification 54C08, 54H05.

\section{Introduction}

For a set $X$, a fuzzy set in $X$ is a function $\lambda: X \rightarrow[0.1]$. Here $\lambda(x)$ represents the degree of membership of $x$ in the fuzzy subset $A$ of $X$ and by $\chi_{A}$, we mean the fuzzy set that maps every element in $A$ to 1 and every element outside $A$ to 0 . Fuzzy topological spaces (simply, spaces) were first introduced by $[1,2]$. A fuzzy topology on a set $X$ is a collection $\mathfrak{T}$ of subsets of $X$ satisfying: $0,1 \in \mathfrak{T}$, is closed under formation of finite intersections and is closed under formation of arbitrary unions. Fuzzy topological spaces were studied by several authors, see for example $[1,3-8]$. Let $(X, \mathfrak{T})$ be a fuzzy topological space. If $\lambda$ is a fuzzy set, then the closure of $\lambda$ (the smallest fuzzy closed set containing $\lambda$ ) and the interior of $\lambda$ (the largest fuzzy open set in $\lambda$ ) will be denoted by $C l_{\mathfrak{T}}(\lambda)$ and $\operatorname{Int}_{\mathfrak{T}}(\lambda)$, respectively. If no ambiguity appears, we use

$$
\bar{\lambda} \text { and } \stackrel{o}{\lambda}
$$

instead, respectively. A fuzzy set $\lambda$ is called fuzzy semiopen [6] if there exists a fuzzy open set $\mu$ such that $\mu \leq \lambda \leq C l_{\mathfrak{T}}(\mu)$. Clearly $\lambda$ is a fuzzy semiopen set if and only if $\lambda \leq C l_{\mathfrak{T}}\left(\operatorname{Int}_{\mathfrak{T}}(\lambda)\right)$. A complement of a fuzzy semiopen set is called fuzzy semiclosed. The fuzzy semi-interior of $\lambda$ is the union of all fuzzy semi-open subsets contained in $\lambda$ and is denoted by $\operatorname{sInt}(\lambda) \cdot \lambda$ is called fuzzy preopen if $\lambda \leq \operatorname{Int}_{\mathfrak{T}}\left(C l_{\mathfrak{T}}(\lambda)\right) \cdot \lambda$ is called fuzzy $\alpha$-open if $\lambda \leq \operatorname{Int}_{\mathfrak{T}}\left(C l_{\mathfrak{T}}\left(\operatorname{Int}_{\mathfrak{T}}(\lambda)\right)\right)$ and fuzzy $\beta$-open if $\lambda \leq C l_{\mathfrak{T}}\left(\operatorname{Int}_{\mathfrak{T}}\left(C l_{\mathfrak{T}}(\lambda)\right)\right) . \quad \lambda$ is called fuzzy regular open if $\lambda=\operatorname{Int}_{\mathfrak{T}}\left(C l_{\mathfrak{T}}(\lambda)\right)$. Complements of fuzzy regular open sets are called fuzzy regular closed. $\lambda$ is called fuzzy preclosed if $C l(\operatorname{Int}(\lambda)) \leq \lambda$ and fuzzy regular closed if $\lambda=C l(\operatorname{Int}(\lambda)) . \quad \lambda$ is a fuzzy generalized closed set if $\lambda \leq \mu$ and $\mu \in \mathfrak{T}$ implies that $\lambda \leq \mu$. A fuzzy function $f:(X, \mathfrak{T}) \rightarrow\left(Y, \mathfrak{T}^{\prime}\right)$ is called fuzzy generalized continuous if $f^{-1}(\lambda)$ is fuzzy generalized closed in $(X, \mathfrak{T})$ for every fuzzy closed set $\lambda$ of $\left(Y, \mathfrak{T}^{\prime}\right)$ and fuzzy contrasemi-continuous if $f^{-1}(\lambda)$ is fuzzy semiopen in $(X, \mathfrak{T})$ for every fuzzy closed set $\lambda$ of $\left(Y, \mathfrak{T}^{\prime}\right)$. For more on the preceding notions, the reader is referred to $[1,2,4,6,8]$.

We introduce the relatively new notions of fuzzy L-closed sets, which is closely related to the class of fuzzy closed subsets. We show that the collection of all fuzzy L-open 
subsets of a space $(X, \mathfrak{T})$ forms a fuzzy topology that is finer than $\mathfrak{T}$ and we investigate several characterizations of fuzzy L-open and fuzzy L-closed notions via the operations of interior and closure. In section 3, we introduce the notion of fuzzy L-generalized closed sets and study connections to other weak and strong forms of fuzzy generalized closed sets. In addition several interesting properties and constructions of fuzzy L-generalized closed sets are discussed. Section 4 is devoted to introducing and studying fuzzy L-generalized continuous and fuzzy L-generalized irresolute functions and connections to other similar forms of fuzzy continuity.

\section{Fuzzy L-closed sets}

We begin this section by introducing the notions of fuzzy L-open and fuzzy L-closed subsets.

Definition 1 Let $\mu$ be a fuzzy subset of a space $(X, \tau)$. The fuzzy L-interior of $\mu$ is the union of all fuzzy subsets of $X$ whose closures are contained in Int $(\mu)$, and is denoted by $\operatorname{Int}_{L}(\mu)$. The fuzzy $L$-closure of $\mu$ is $C l_{L}(\mu)$ is the smallest fuzzy closed set containing $\mu$. $\mu$ is called fuzzy L-open if $\mu=\operatorname{Int}_{L}(\mu)$. The complement of a fuzzy L-open subset is called fuzzy L-closed.

Clearly $\operatorname{Int}_{L}(\mu) \leq \operatorname{Int}(\mu) \leq \mu$ and hence every fuzzy L-open set is fuzzy open and thus every fuzzy L-closed set is fuzzy closed, but the converses needs not be true.

Example 1 Let $X=\{a, b, c\}$ and $\tau=\left\{0,1, \chi_{\{a\}}, \chi_{\{b\}}, \chi_{\{a, b\}}\right\}$. Then $\chi_{\{a, c\}}$ is a fuzzy open set that is not a fuzzy L-open as $\operatorname{Int}_{L}\left(\operatorname{Int}_{L}\left(\chi_{\{a, c\}}\right)=0\right.$.

Next, we show that the collection of all fuzzy L-open subsets of a space $(X, \tau)$ forms a fuzzy topology $\tau_{F L}$ that is finer than $\tau$.

Theorem 1 If $(X, \tau)$ is a fuzzy space, then $\left(X, \tau_{F L}\right)$ is a fuzzy space such that $\tau \supseteq \tau_{F L}$.

Proof We only need to show $\left(X, \tau_{F L}\right)$ is a fuzzy space. Clearly 0 and 1 are fuzzy L-open sets. If $\mu, \gamma \in \tau_{F L}$, then $\mu=\operatorname{Int}_{L}(\mu)$ and $\gamma=\operatorname{Int}_{L}(\gamma)$. Now $\operatorname{Int}_{L}(\mu \cap \gamma)=\vee\{\theta \in \tau: C l(\theta) \leq$ $\operatorname{Int}(\mu \wedge \gamma)\}=\vee\{\theta \in \tau: C l(\theta) \leq \operatorname{Int}(\mu) \wedge \operatorname{Int}(\gamma)\}$. Thus $\operatorname{Int}_{L}(\mu \wedge \gamma) \geq \operatorname{Int}_{L}(\mu) \wedge \operatorname{Int}_{L}(\gamma)=$ $\mu \wedge \gamma$. Therefore, $\mu \wedge \gamma=\operatorname{Int}_{L}(\mu \wedge \gamma)$ and so $\mu \wedge \gamma \in \tau_{F L}$.

If $\left\{\mu_{\alpha}: \alpha \in \Delta\right\}$ is a collection of fuzzy L-open subsets of $X$, then for every $\alpha \in \Delta$, $\operatorname{Int}_{L}(\mu \alpha)=\mu_{\alpha}$. Hence

$$
\begin{aligned}
\operatorname{Int}_{L}\left(\vee_{\alpha \in \Delta} \mu \alpha\right) & =\vee\left\{\theta \in \tau: C l(\theta) \leq \operatorname{Int}\left(\vee_{\alpha \in \Delta} \mu \alpha\right)\right\} \\
& \geq \vee\left\{\theta \in \tau: C l(\theta) \leq \vee_{\alpha \in \Delta} \operatorname{Int}(\mu \alpha)\right\} \\
& \geq \vee\{\theta \in \tau: C l(\theta) \leq \mu \alpha\} \text { for every } \alpha \in \Delta \\
& =\operatorname{Int}_{L}(\mu \alpha) \text { for every } \alpha \in \Delta \\
& =\mu \alpha \text { for every } \alpha \in \Delta .
\end{aligned}
$$

Hence $\vee_{\alpha \in \Delta} \mu \alpha \leq \operatorname{Int}_{L}\left(\vee_{\alpha \in \Delta} \mu \alpha\right)$ and thus $\vee_{\alpha \in \Delta} \mu \alpha$ is fuzzy L-open.

In classical topology, a set is always contained in its closure, but this is not the case in $\tau_{F L}$. Next we show that $\mu \leq C l_{L}(\mu)$ needs not be true.

Example 2 Let $X=\{a, b, c, d\}$ and $\tau=\left\{0,1, \chi_{\{a\}}, \chi_{\{a, b\}}, \chi_{\{c, d\},} \chi_{\{a, c, d\}}\right\}$. Then $\chi_{\{c\}} \leq$ $\chi_{\{a, b, c\}}$, but $\chi_{\{c\}} \not \leq C l_{F L}\left(\chi_{\{a, b, c\}}\right)$. 
One might think that a fuzzy subset $\mu$ of a fuzzy space $X$ is fuzzy L- closed if and only if $\mu=C l_{L}(\mu)$, but this is not true as shown in the next example.

Example 3 Consider the space in Example 2 and cosider $\mu=\chi_{\{b, c\}}$. Since $C l\left(\chi_{\{a\}}\right)=$ $\chi_{\{a, c\}}, \chi_{\{a\}} \not \leq C l_{L}(\mu)$. Since $C l_{L}(\mu)=\mu$, but $\mu$ is not a fuzzy L-open set.

Lemma 1 For any fuzzy set $\mu$ of a fuzzy space $X$,

(i) $\operatorname{Int}(\mu) \leq C l_{L}(\mu)$.

(ii) $\operatorname{Int}(\mu)=0$ if and only if $C l_{L}(\mu)=0$.

Proof

(i) $\lambda \not \subset C l_{L}(\mu)$ implies that there exists a fuzzy open set $\theta$ containing $\lambda$ such that $C l(\theta) \wedge$ $\operatorname{Int}(\mu)=0$. Hence $\lambda \not \leq \operatorname{Int}(\mu)$.

(ii) If $\lambda \leq C l_{L}(\mu)$, then for every fuzzy open subset $\theta$ containing $\lambda, C l(\theta) \wedge \operatorname{Int}(\mu) \neq 0$. Hence there exists $\gamma \leq C l(\theta) \wedge \operatorname{Int}(\mu)$ and as $\operatorname{Int}(\mu)$ is fuzzy open, $\theta \wedge \operatorname{Int}(\mu) \neq 0$. Therefore $\operatorname{Int}(\mu) \neq 0$.

Conversely if $C l_{L}(\mu)=0$, then by (i) as $\operatorname{Int}(\mu) \leq C l_{L}(\mu), \operatorname{Int}(\mu)=0$.

Lemma 2 The union of a fuzzy open set with a fuzzy L-open-set is fuzzy open.

Proof Let $\mu$ be an open set and $\eta$ be a fuzzy L-open set. For all $\gamma \leq \mu \vee \eta, \gamma \leq \mu$ or $\gamma \leq \eta$ and so $\gamma \leq \operatorname{Int}(\mu) \leq \operatorname{Int}(\mu \vee \eta)$ or $\gamma \leq \operatorname{Int}_{L}(\eta) \leq \operatorname{Int}_{L}(\mu \vee \eta) \leq \operatorname{Int}(\mu \vee \eta)$

Corollary 1 The intersection of a fuzzy closed set with a fuzzy L-closed set is fuzzy closed.

Lemma 3 If $\lambda$ is a fuzzy semiopen set of a fuzzy space $X, C l_{L}(\mu)=C l(\mu)$.

Proof If $\theta$ is a fuzzy open set containing $\lambda$ such that $C l(\theta) \wedge \operatorname{Int}(\mu) \neq 0$, then there exists $\gamma \leq C l(\theta) \wedge \operatorname{Int}(\mu)$. Thus $\theta \wedge \operatorname{Int}(\mu) \neq 0$ and so $\theta \wedge \mu \neq 0$. Therefore $C l_{L}(\mu) \leq C l(\mu)$.

Conversely if for every fuzzy open set $\theta$ containing $\mu$ we have $\theta \wedge \mu \neq 0, \theta \wedge \operatorname{Int}(C l(\mu)) \neq 0$, since $\mu$ is fuzzy semiopen. Thus there exists $\gamma \leq \theta \wedge \operatorname{Int}(C l(\mu))$ and so $\theta \wedge \operatorname{Int}(\mu) \neq 0$ which implies that $C l(\theta) \wedge \operatorname{Int}(\mu) \neq 0$. Hence $C l(\mu) \leq C l_{L}(\mu)$.

\section{Corollary 2}

(i) For any fuzzy subset $\mu$ of $X, C l_{L}(\mu) \leq C l(\mu)$.

(ii) If $\mu$ is a fuzzy semiopen subset of a space $X$, then $\mu \leq C l_{L}(\mu)$.

Lemma 4 If $\mu$ is a fuzzy L-closed set in a fuzzy space $X$, then $C l_{L}(\mu) \leq \mu$.

Proof If $\mu$ is a fuzzy L-closed subset, then $\mu$ is fuzzy closed and thus by Corollary 2 (i), $C l_{L}(\mu) \leq \mu$.

Next, we show that a fuzzy preclosed set that is also fuzzy semiopen equals its fuzzy L-closure.

Theorem 2 If $\mu$ is a fuzzy regular closed subset of a fuzzy space $X$, then $C l_{L}(\mu) \leq \mu$.

Proof $C l_{L}(\mu) \leq C l(\mu) \leq C l(C l(\operatorname{Int}(\mu)))=C l(\operatorname{Int}(\mu)) \leq \mu$. This together with Corollary 2 implies that $\mu=C l_{L}(\mu)$. 


\section{$3 \quad$ Fuzzy L-generalized closed sets}

In this section, we introduce the notion of fuzzy L-generalized closed set. Moreover, several interesting properties and constructions of these subsets are discussed.

Definition 2 A fuzzy subset $\mu$ of a fuzzy space $X$ is called fuzzy L-generalized closed set if whenever $\theta$ is a fuzzy open subset containing $\mu$, we have $C l_{L}(\mu) \leq \theta$. $\mu$ is fuzzy L-generalized open if $1-\mu$ is fuzzy L-generalized closed set.

Theorem 3 A subset $\mu$ of $(X, \tau)$ is fuzzy L-generalized open if and only if $\eta \leq \operatorname{Int}_{L}(\mu)$, whenever $\eta \leq \mu$ and $\eta$ is fuzzy closed in $(X, \tau)$.

Proof Let $\mu$ be a fuzzy L-generalized open set and $\eta$ be a fuzzy closed subset such that $\eta \leq \mu$. Then $1-\mu \leq 1-\eta$. As $1-\mu$ is fuzzy L-generalized closed set and as $1-\eta$ is fuzzy open, $C l_{L}(1-\mu) \leq 1-\eta$. So $\eta \leq 1-C l_{L}(1-\mu)=\operatorname{Int}_{L}(\mu)$.

Conversely if $1-\mu \leq \theta$ where $\theta$ is fuzzy open, then the fuzzy closed set $1-\theta \leq \mu$. Thus $1-\theta \leq \operatorname{Int}_{L}(\mu)=1-C l_{L}(1-\mu)$ and so $C l_{L}(1-\mu) \leq \theta$.

Next we show that every fuzzy L- closed set is fuzzy L- generalized closed . Moreover, the class of fuzzy generalized closed sets is properly placed between the classes of fuzzy semiopen sets that are fuzzy closed and fuzzy L-generalized closed sets. Clearly every fuzzy closed set that is fuzzy semiopen, by Lemma 1, is a fuzzy L- closed set. A fuzzy closed set is trivially fuzzy generalized closed and every fuzzy generalized closed set is fuzzy L-generalized closed by Corollary 2 (i).

The following result follows from Corollary 2 (i) and the fact that every fuzzy L-closed set is fuzzy closed:

Lemma 5 Every fuzzy L-closed-set is fuzzy L-generalized closed.

The converse of the preceding result needs not be true.

Example 4 Let $X=\{a, b, c, d\}$ and $\tau=\left\{0,1, \chi_{\{b\}}, \chi_{\{c\}}, \chi_{\{b, c\}}, \chi_{\{a, b\}}, \chi_{\{a, b, c\}}\right\}$. Then as $C l_{L}\left(\chi_{\{a\}}\right)=0, \chi_{\{a\}}$ is fuzzy L-generalized closed, but it is not fuzzy L-closed and not fuzzy generalized closed and hence not fuzzy closed. Also $\chi_{\{b, d\}}$ is an fuzzy generalized closed set that is not fuzzy closed.

The following is an immediate result from Lemma 1:

Theorem 4 If $\mu$ is a fuzzy semiopen subset of a space $X$, the following are equivalent:

(1) $\mu$ is fuzzy L-generalized closed;

(2) $\mu$ is fuzzy generalized closed.

Its clear that if $\mu \leq \gamma$, then $\operatorname{Int}_{L}(\mu) \leq \operatorname{Int}_{L}(\gamma)$ and $C l_{L}(\mu) \leq C l_{L}(\gamma)$.

Lemma 6 If $\mu$ and $\gamma$ are fuzzy sets in a fuzzy space $X$, then $C l_{L}(\mu) \vee C l_{L}(\gamma)=C l_{L}(\mu \vee \gamma)$ and $C l_{L}(\mu \wedge \gamma) \leq C l_{L}(\mu) \wedge C l_{L}(\gamma)$.

Proof Since $\mu$ and $\gamma$ are subsets of $\mu \vee \gamma, C l_{L}(\mu) \vee C l_{L}(\gamma) \leq C l_{L}(\mu \vee \gamma)$. On the other hand, if $\eta \leq C l_{L}(\mu \vee \gamma)$ and $\theta$ is a fuzzy open set containing $\eta$, then $C l(\theta) \wedge \operatorname{Int}(\mu \vee \gamma) \neq 0$. Hence either $C l(\theta) \wedge \operatorname{Int}(\mu) \neq 0$ or $C l(\theta) \wedge \operatorname{Int}(\gamma) \neq 0$. Thus $\eta \leq C l_{L}(\mu) \vee C l_{L}(\gamma)$.

Finally since $\mu \wedge \gamma$ is a fuzzy subset of $\mu$ and $\gamma, C l_{L}(\mu \wedge \gamma) \leq C l_{L}(\mu) \wedge C l_{L}(\gamma)$.

Corollary 3 Finite union of fuzzy L-generalized closed sets is fuzzy L-generalized closed.

While the finite intersection of fuzzy L-generalized closed sets needs not be fuzzy Lgeneralized closed. 


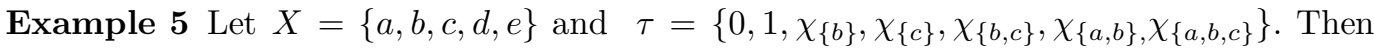
$\lambda=\chi_{\{a, c, d\}}$ and $\mu=\chi_{\{b, c, e\}}$ are fuzzy L-generalized closed sets since the only super fuzzy open set of both is 1 . But $\lambda \wedge \mu=\chi_{\{c\}}$ is not fuzzy L-generalized closed.

Theorem 5 The intersection of a fuzzy L-generalized closed set with a fuzzy L-closed set is fuzzy L-generalized closed.

Proof Let $\mu$ be a fuzzy L-generalized closed set and $\eta$ be a fuzzy L-closed set. Let $\theta$ be a fuzzy open set containing $\mu \wedge \eta$. Then $\mu \leq \theta \vee 1-\eta$. Since $1-\eta$ is fuzzy L-open, by Lemma $3, \theta \vee 1-\eta$ is fuzzy open and since $\mu$ is fuzzy L-generalized closed set, $C l_{L}(\mu \wedge \eta) \leq$ $C l_{L}(\mu) \wedge C l_{L}(\eta)$ and by Lemma $6, C l_{L}(\mu \wedge \eta) \leq C l_{L}(\mu) \wedge \eta \leq(\theta \vee 1-\eta) \wedge \eta=\theta \wedge \eta \leq \theta . \square$

\section{Fuzzy L-generalized continuous and fuzzy L-generalized irreso- lute functions}

Definition 3 A fuzzy function $f:(X, \tau) \rightarrow\left(Y, \tau^{\prime}\right)$ is called

(1) Fuzzy L-generalized continuous if $f^{-1}(\lambda)$ is fuzzy L-generalized closed set in $(X, \tau)$ for every fuzzy closed set $\lambda$ of $\left(Y, \tau^{\prime}\right)$,

(2) Fuzzy L-generalized irresolute if $f^{-1}(\lambda)$ is fuzzy L-generalized closed set in $(X, \tau)$ for every fuzzy L-generalized closed set set $\lambda$ of $\left(Y, \tau^{\prime}\right)$.

Lemma 7 Let $f:(X, \tau) \rightarrow\left(Y, \tau^{\prime}\right)$ be a fuzzy generalized continuous. Then $f$ is fuzzy $L$-generalized continuous, but not conversely.

Proof Follows from the fact that every fuzzy generalized closed set is fuzzy L-generalized closed.

Example 6 Let $X=\{a, b, c, d, e\}$ and $\tau=\left\{0,1, \chi_{\{b\}}, \chi_{\{c\}}, \chi_{\{b, c\}}, \chi_{\{a, b\}}, \chi_{\{a, b, c\}}\right\}$ and $\tau^{\prime}=\left\{0,1, \chi_{\{d\}}\right\}$. Let $f:(X, \tau) \rightarrow\left(X, \tau^{\prime}\right)$ be the identity function. Since $f^{-1}\left(\chi_{\{a, b, c\}}\right)=$ $\chi_{\{a, b, c\}}=C l_{L}\left(\chi_{\{a, b, c\}}\right), f$ is fuzzy L-generalized continuous, but $f$ is not fuzzy generalized continuous and hence not fuzzy continuous.

Even the composition of fuzzy L-generalized continuous functions needs not be fuzzy L-generalized continuous.

Example 7 Let $f$ be the fuzzy function in Example 6 and $g:\left(X, \tau^{\prime}\right) \rightarrow\left(X, \tau^{\prime \prime}\right)$ be the identity fuzzy function. It is easy to see that $g$ is also a fuzzy L-generalized continuous function, but $g \circ f$ is not fuzzy L-generalized continuous as $\chi_{\{c\}}$ is fuzzy closed in $\left(X, \tau^{\prime \prime}\right)$, but not fuzzy L-generalized continuous in $(X, \tau)$.

Corollary 4 If $f:(X, \tau) \rightarrow\left(Y, \tau^{\prime}\right)$ is a fuzzy continuous and fuzzy contra-semi-continuous, then $f$ is fuzzy L-generalized continuous.

Proof If $\lambda$ is a fuzzy closed subset of $Y$, then as $f$ is fuzzy continuous $f^{-1}(\lambda)$ is fuzzy closed and as $f$ is fuzzy contra-semi-continuous, $f^{-1}(\lambda)$ is fuzzy semiopen. Thus $f^{-1}(\lambda)$ is fuzzy L-generalized closed set.

We end this section by giving a necessary condition for a fuzzy L-generalized irresolute function to be fuzzy L-generalized continuous.

Theorem 6 If $f:(X, \tau) \rightarrow\left(Y, \tau^{\prime}\right)$ is bijective, fuzzy open and fuzzy L-generalized irresolute, then $f$ is fuzzy L-generalized closed. 
Proof Let $\lambda$ be a fuzzy closed subset of $Y$ and let $f^{-1}(\lambda) \leq \gamma$, where $\gamma \in \tau$. Clearly, $\lambda \leq f(\gamma)$. Since $f(\gamma) \in \tau^{\prime}$ and since $\lambda$ is fuzzy L-generalized closed set, $C l_{L}(\lambda) \leq f(\gamma)$ and thus $f^{-1}\left(C l_{L}(\lambda)\right) \leq \gamma$. Since $f$ is fuzzy L-generalized irresolute and since $C l_{L}(\lambda)$ is fuzzy L-generalized closed set in $Y, f^{-1}\left(C l_{L}(\lambda)\right.$ is fuzzy L-generalized closed set. $f^{-1}\left(C l_{L}(\lambda) \leq\right.$ $C l_{L}\left(f^{-1}\left(C l_{L}(\lambda)\right)\right)=f^{-1}\left(C l_{L}(\lambda)\right) \leq \gamma$. Therefore, $f^{-1}(\lambda)$ is fuzzy L-generalized closed set and hence, $f$ is fuzzy L-generalized continuous.

\section{Acknowledgemnts}

The authors would like to thank the referees for useful comments and suggestions.

\section{References}

[1] Chakrabarty, M. K. and Ahsanullah, T. M. Fuzzy topology on fuzzy sets and tolerance topology. Fuzzy Set Syst. 1992. 45: 103-108.

[2] Chang, C. L. Fuzzy topological spaces. J. Math. Anal. Appl. 1968. 24: 182-190.

[3] Al-Hawary, T. Fuzzy $\omega_{0}$-open sets. Bull. Korian Math. Soc. 2008. 45(4): 749-755.

[4] Chaudhuri, A. K. and Das, P. Some results on fuzzy topology on fuzzy sets. Fuzzy Set Syst. 1993. 56: 331-336.

[5] Haydar Es, A. Almost compactness and near compactness in fuzzy topological spaces. Fuzzy Sets and Syst. 1987. 22: 289-295.

[6] Mahmoud, F. S. Fath Alla, M. A. and Abd Ellah, S. M. Fuzzy topology on fuzzy sets: fuzzy semicontinuity and fuzzy semiseparation axioms. Appl. Math. Comput. 2004. 153: $127-140$.

[7] Wong, C. K. Covering properties of fuzzy topological spaces, J. Math. Anal. Appl. 43(1973), 697-704.

[8] Wong, C. K. Fuzzy points and local properties of fuzzy topology. J. Math. Anal. Appl. 1974. 46: 316-328. 